\title{
Super-leading logarithms in non-global observables in QCD: colour basis independent calculation
}

\author{
J.R. Forshaw and A. Kyrieleis \\ School of Physics \& Astronomy, University of Manchester, \\ Oxford Road, Manchester M13 9PL, U.K. \\ E-mail: forshaw@mail.cern.ch, kyrieleis@hep.man.ac.ul
}

\section{M.H. Seymour}

School of Physics 85 Astronomy, University of Manchester, Oxford Road, Manchester M13 9PL, U.K., and

Theoretical Physics Group (PH-TH), CERN,

CH-1211 Geneva 23, Switzerland

E-mail: mike.seymour@cern.ch

\begin{abstract}
In a previous paper we reported the discovery of super-leading logarithmic terms in a non-global QCD observable. In this short update we recalculate the first superleading logarithmic contribution to the 'gaps between jets' cross-section using a colour basis independent notation. This sheds light on the structure and origin of the super-leading terms and allows them to be calculated for gluon scattering processes for the first time.
\end{abstract}

KeYwords: Jets, Hadronic Colliders, QCD. 


\section{Contents}

1. Introduction 1

2. Summary of previous calculations 2

3. Colour basis independent notation 6

4. One gluon outside the gap 10

5. Results 16

6. Outlook 17

\section{Introduction}

In perturbative calculations of the cross-section for the production of a pair of jets with a rapidity gap between them, it is often assumed that the observable is fully inclusive outside the gap region and therefore that there is a perfect real-virtual cancellation there [1]-[6]. In [7] we made a calculation of the first correction to this picture coming from one (real or virtual) gluon being emitted outside the gap and dressed by an arbitrary number of additional virtual gluons. ${ }^{1}$ Based on the work of [8] -10 , we expected to find an additional tower of leading logarithms, known as non-global logarithms, generated by the fact that gluons outside the gap are prevented from radiating into the gap region by the gap requirement. At leading order this is an edge effect: gluons just outside the gap are suppressed by the non-emission just inside the gap, leading to the existence of a 'buffer zone' in all-orders calculations [9].

However, we were surprised to find an additional long-range source of mis-cancellation that leads to a tower of super-leading logarithms. We traced their origin to the imaginary parts of the loop integrals, sometimes known as the Coulomb gluon contributions. We view this as being due to a breakdown of naive coherence for initial-state radiation [11, 12]. A similar conclusion was also reached, for a different process, in 13, 14.

Although we expect that our conclusions are valid for any QCD scattering process, our calculation was only actually performed for the specific case of quark-quark scattering, $q q \rightarrow q q$. Generalizing this to other $2 \rightarrow 2$ scattering processes, let alone the general $2 \rightarrow n$ case, is troublesome due to the large dimensionality of the colour bases in which the anomalous dimension matrices need to be calculated. Diagrammatic approaches 115 do not require an explicit colour basis and do not get any more complicated when replacing the

\footnotetext{
${ }^{1}$ We explain more precisely what we mean by a virtual gluon being 'inside' or 'outside' the gap below.
} 
quarks by gluons, but their disadvantage is that the number of cut diagrams soon becomes prohibitively large.

Historically, in discussions that may turn out to be quite relevant to the present case [16], a significant advantage was achieved by performing the calculation in a colour basis independent way. The purpose of the present paper is to repeat the calculation of [7] in the colour basis independent notation. Of course the results agree with those of [7], but they are obtained more transparently, and in a way that generalizes easily to other processes. We therefore believe that they add considerably to the understanding of the origin and structure of the super-leading logarithms, although they do nothing to solve the problem of how to deal with them in general.

We view the present paper as an addendum to [7], and therefore do not repeat the introduction, motivation or discussion of numerical results that are contained there. We begin in section 2 with a brief summary of the previous calculations, before introducing the colour basis independent notation in section 3. In section t we use it to recalculate the leading non-zero contribution to the 'one gluon outside the gap' cross-section and show that it boils down to the sum over a relatively small number of Feynman diagrams. The method also enables us to calculate, for the first time, the coefficient of the first superleading logarithm for gluon scattering processes and this is done in section 5. Finally, in section 6, we make a brief outlook. Sections 4 and 5 contain new results, while sections 2 and 3 are important to provide a pedagogical introduction to the calculation.

\section{Summary of previous calculations}

The main non-trivial aspect when calculating gap cross-sections in hadron collisions is the fact that the hard scattering matrix elements have a non-trivial colour structure. The simplest case, $q q \rightarrow q q$, for example, has two independent colour structures. These form a vector space and for a concrete calculation we can introduce a basis for this space. In this example, we take the $t$-channel basis in which the two colour structures correspond to the exchange of a singlet or an octet in the $t$-channel. In order to anticipate the structure of the colour basis independent calculation, we choose to normalize our colour states, which will mean that the soft matrix $\mathbf{S}_{V}$, defined in [7], is equal to the identity matrix. This is the only difference in notation between this section and [7]. With this normalization, the colour basis states for $q_{i} q_{j} \rightarrow q_{k} q_{l}$ are

$$
\begin{aligned}
& \mathbf{C}_{1} \equiv \frac{1}{N} \delta_{k i} \delta_{l j}, \\
& \mathbf{C}_{8} \equiv \frac{2}{\sqrt{N^{2}-1}} T_{k i}^{a} T_{l j}^{a},
\end{aligned}
$$

where $N$ is the number of colours.

In general, to make an all-orders resummed calculation in this process, one needs to calculate the contribution from an arbitrary number of additional virtual or real gluons. Virtual gluons do not change the dimensionality of the colour space, but real gluons do: each emitted gluon takes us to a higher-dimensional space, so the fully general all-orders 
calculation becomes intractable. However, one can make a series of steps to reformulate the all-orders calculation as an evolution of the original $2 \rightarrow 2$ process in colour space. We start by outlining the thinking underlying the original calculations.

1. To extract the leading logarithms of $Q / Q_{0}\left(Q\right.$ is the hard scattering scale and $Q_{0}$ is the veto scale used to define the gap) one can make a strong ordering approximation. The calculation of the $n$-loop correction to the $2 \rightarrow 2$ process therefore nests and one can apply the 1-loop correction, with the loop gluon attached only to the external partons, $n$ times, giving an exponentiating structure.

2. One can reduce the dimensionality of the loop integral by one by integrating over (e.g.) the energy. The corresponding contour integral contains two kinds of pole: one where the exchanged gluon goes on shell (which we refer to as the eikonal gluon contribution) and one where the external partons go on shell (which we refer to as the Coulomb gluon contribution). The former has exactly the same structure as the phase-space integral for emission of a real gluon; the latter does not.

3. It is easy to show that for a fully-inclusive observable, the eikonal gluon contribution is exactly equal and opposite to the real gluon emission one. In conventional calculations, it is assumed that the observable is sufficiently inclusive for this cancellation to be maintained for all emissions 'outside the gap'.

4. As a result of step 3, real gluon emission does not contribute since outside the gap (and below threshold within the gap) we have assumed a perfect real-virtual cancellation whilst inside the gap and above threshold, real emission is forbidden since it spoils the gap definition.

Under these four assumptions, the all-orders calculation involves calculating only virtual gluon corrections with the eikonal gluons integrated over the gap region. One finds that

$$
\sigma=\mathbf{M}^{\dagger} \mathbf{M}
$$

with

$$
\mathbf{M}=\left(\begin{array}{l}
M^{(1)} \\
M^{(8)}
\end{array}\right)
$$

The colour evolution is given by

$$
\mathbf{M}\left(Q_{0}\right)=\exp \left(-\frac{2 \alpha_{s}}{\pi} \int_{Q_{0}}^{Q} \frac{d k_{T}}{k_{T}} \boldsymbol{\Gamma}\right) \mathbf{M}(Q),
$$

with boundary condition

$$
\mathbf{M}(Q) \equiv \mathbf{M}_{0}=\left(\begin{array}{c}
0 \\
\sqrt{\sigma_{\text {born }}}
\end{array}\right)
$$

and anomalous dimension matrix

$$
\boldsymbol{\Gamma}=\left(\begin{array}{cc}
\frac{N^{2}-1}{4 N} \rho(Y, \Delta y) & \frac{\sqrt{N^{2}-1}}{2 N} i \pi \\
\frac{\sqrt{N^{2}-1}}{2 N} i \pi & -\frac{1}{N} i \pi+\frac{N}{2} Y+\frac{N^{2}-1}{4 N} \rho(Y, \Delta y)
\end{array}\right) .
$$


Note that, as pointed out in [17] and proved in [18], $\Gamma$ is symmetric in this, normalized, basis. The function $\rho$ appearing here is defined in [7]: it is small in the region of interest (large $Y$ ) and not very relevant to the present discussion. Note that (2.5) includes the $i \pi$ terms generated by Coulomb gluon emissions only at $k_{T}>Q_{0}$. The contribution from these virtual corrections below $Q_{0}$ does not cancel but instead exponentiates to produce a net phase in the amplitude that does not contribute to the cross-section.

The aim of the present calculation is to check the validity of the assumption articulated in step 3 above, by calculating the correction coming from allowing one gluon outside the gap, $\sigma_{1}$. This can be written as the sum of a real contribution plus an eikonal virtual contribution, ${ }^{2}$ each integrated over the phase space region outside the gap and each dressed with any number of Coulomb gluons or in-gap eikonal gluons. Thus

$$
\sigma_{1}=-\frac{2 \alpha_{s}}{\pi} \int_{Q_{0}}^{Q} \frac{d k_{T}}{k_{T}} \int_{\text {out }} \frac{d y d \phi}{2 \pi}\left(\Omega_{R}+\Omega_{V}\right)
$$

where

$$
\begin{aligned}
\Omega_{V}=\mathbf{M}_{0}^{\dagger} \exp \left(-\frac{2 \alpha_{s}}{\pi} \int_{Q_{0}}^{Q} \frac{d k_{T}^{\prime}}{k_{T}^{\prime}} \boldsymbol{\Gamma}^{\dagger}\right) \\
\quad \times \exp \left(-\frac{2 \alpha_{s}}{\pi} \int_{Q_{0}}^{k_{T}} \frac{d k_{T}^{\prime}}{k_{T}^{\prime}} \boldsymbol{\Gamma}\right) \gamma \exp \left(-\frac{2 \alpha_{s}}{\pi} \int_{k_{T}}^{Q} \frac{d k_{T}^{\prime}}{k_{T}^{\prime}} \boldsymbol{\Gamma}\right) \mathbf{M}_{0}+\text { c.c., } \\
\Omega_{R}=\mathbf{M}_{0}^{\dagger} \exp \left(-\frac{2 \alpha_{s}}{\pi} \int_{k_{T}}^{Q} \frac{d k_{T}^{\prime}}{k_{T}^{\prime}} \boldsymbol{\Gamma}^{\dagger}\right) \mathbf{D}_{\mu}^{\dagger} \exp \left(-\frac{2 \alpha_{s}}{\pi} \int_{Q_{0}}^{k_{T}} \frac{d k_{T}^{\prime}}{k_{T}^{\prime}} \boldsymbol{\Lambda}^{\dagger}\right) \\
\quad \times \exp \left(-\frac{2 \alpha_{s}}{\pi} \int_{Q_{0}}^{k_{T}} \frac{d k_{T}^{\prime}}{k_{T}^{\prime}} \boldsymbol{\Lambda}\right) \mathbf{D}^{\mu} \exp \left(-\frac{2 \alpha_{s}}{\pi} \int_{k_{T}}^{Q} \frac{d k_{T}^{\prime}}{k_{T}^{\prime}} \boldsymbol{\Gamma}\right) \mathbf{M}_{0}
\end{aligned}
$$

The two contributions have a common evolution from $Q$ down to $k_{T}$, followed by, for the virtual contribution, a virtual eikonal emission at scale $k_{T}$,

$$
\gamma=\frac{1}{2}\left(\begin{array}{cc}
\frac{N^{2}-1}{2 N}\left(\omega_{13}+\omega_{24}\right) & \frac{\sqrt{N^{2}-1}}{2 N}\left(-\omega_{12}-\omega_{34}+\omega_{14}+\omega_{23}\right) \\
\frac{\sqrt{N^{2}-1}}{2 N}\left(-\omega_{12}-\omega_{34}+\omega_{14}+\omega_{23}\right) & \frac{N}{2}\left(\omega_{14}+\omega_{23}\right)-\frac{1}{2 N}\left(\omega_{13}+\omega_{24}\right) \\
& +\frac{1}{N}\left(\omega_{12}+\omega_{34}-\omega_{14}-\omega_{23}\right)
\end{array}\right),
$$

which can appear on either side of the cut, with

$$
\omega_{i j}=\frac{1}{2} k_{T}^{2} \frac{p_{i} \cdot p_{j}}{\left(p_{i} \cdot k\right)\left(p_{j} \cdot k\right)},
$$

followed by further evolution from $k_{T}$ down to $Q_{0}$. The real emission contribution on the other hand involves the matrix $\mathbf{D}^{\mu}$, which describes the emission of a gluon with Lorentz index $\mu$ and is rectangular, being the transformation from the 2-dimensional colour space of $q q \rightarrow q q$ to the 4-dimensional colour space of $q q \rightarrow q q g$. We again work in a basis for the process $q_{i} q_{j} \rightarrow q_{k} q_{l} g_{a}$ that differs from the one in [7] only in its normalization:

$$
\begin{aligned}
\mathbf{C}_{1} & =\frac{1}{\sqrt{N\left(N^{2}-1\right)}}\left(T_{k i}^{a} \delta_{l j}+T_{l j}^{a} \delta_{k i}\right), \\
\mathbf{C}_{2} & =\frac{2 \sqrt{N}}{\sqrt{\left(N^{2}-1\right)\left(N^{2}-4\right)}}\left(T_{k i}^{b} T_{l j}^{c} d^{a b c}\right),
\end{aligned}
$$

\footnotetext{
${ }^{2}$ It makes no sense to speak of Coulomb gluons being in or out of the gap.
} 


$$
\begin{aligned}
& \mathbf{C}_{3}=\frac{1}{\sqrt{N\left(N^{2}-1\right)}}\left(T_{k i}^{a} \delta_{l j}-T_{l j}^{a} \delta_{k i}\right), \\
& \mathbf{C}_{4}=\frac{2}{\sqrt{N\left(N^{2}-1\right)}}\left(T_{k i}^{b} T_{l j}^{c} i f^{a b c}\right) .
\end{aligned}
$$

We then have

$$
\mathbf{D}^{\mu}=\left(\begin{array}{cc}
\frac{\sqrt{N^{2}-1}}{2 \sqrt{N}}\left(-h_{1}^{\mu}-h_{2}^{\mu}+h_{3}^{\mu}+h_{4}^{\mu}\right) & \frac{1}{2 \sqrt{N}}\left(-h_{1}^{\mu}-h_{2}^{\mu}+h_{3}^{\mu}+h_{4}^{\mu}\right) \\
0 & \frac{\sqrt{N^{2}-4}}{2 \sqrt{N}}\left(-h_{1}^{\mu}-h_{2}^{\mu}+h_{3}^{\mu}+h_{4}^{\mu}\right) \\
\frac{\sqrt{N^{2}-1}}{2 \sqrt{N}}\left(-h_{1}^{\mu}+h_{2}^{\mu}+h_{3}^{\mu}-h_{4}^{\mu}\right) & \frac{1}{2 \sqrt{N}}\left(h_{1}^{\mu}-h_{2}^{\mu}-h_{3}^{\mu}+h_{4}^{\mu}\right) \\
0 & \frac{\sqrt{N}}{2}\left(-h_{1}^{\mu}+h_{2}^{\mu}-h_{3}^{\mu}+h_{4}^{\mu}\right)
\end{array}\right),
$$

with

$$
h_{i}^{\mu}=\frac{1}{2} k_{T} \frac{p_{i}^{\mu}}{p_{i} \cdot k} .
$$

Note that $\omega_{i j}=2 h_{i} \cdot h_{j}$ and $\mathbf{D}_{\mu}^{\dagger} \mathbf{D}^{\mu}=-2 \boldsymbol{\gamma}$. Finally, we need the anomalous dimension matrix for the evolution of this five-parton system. This was calculated in [19] and, in the normalized basis, is given by

$$
\begin{aligned}
& \boldsymbol{\Lambda}=\left(\begin{array}{cccc}
\frac{N}{4}(Y-i \pi)+\frac{1}{2 N} i \pi & \frac{\sqrt{N^{2}-4}}{2 N} i \pi & -\frac{N}{4} s_{y} Y & 0 \\
\frac{\sqrt{N^{2}-4}}{2 N} i \pi & \frac{N}{4}(2 Y-i \pi)-\frac{3}{2 N} i \pi & 0 & 0 \\
-\frac{N}{4} s_{y} Y & 0 & \frac{N}{4}(Y-i \pi)-\frac{1}{2 N} i \pi & -\frac{1}{2} i \pi \\
0 & 0 & -\frac{1}{2} i \pi & \frac{N}{4}(2 Y-i \pi)-\frac{1}{2 N} i \pi
\end{array}\right) \\
& +\left(\begin{array}{cccc}
N & 0 & 0 & 0 \\
0 & N & 0 & 0 \\
0 & 0 & N & 0 \\
0 & 0 & 0 & N
\end{array}\right) \frac{1}{4} \rho(Y, 2|y|)+\left(\begin{array}{cccc}
C_{F} & 0 & 0 & 0 \\
0 & C_{F} & 0 & 0 \\
0 & 0 & C_{F} & 0 \\
0 & 0 & 0 & C_{F}
\end{array}\right) \frac{1}{2} \rho(Y, \Delta y) \\
& +\left(\begin{array}{cccc}
-\frac{N}{4} & 0 & -\frac{N}{4} s_{y} & \frac{1}{2} s_{y} \\
0 & -\frac{N}{4} & 0 & \frac{\sqrt{N^{2}-4}}{4} s_{y} \\
-\frac{N}{4} s_{y} & 0 & -\frac{N}{4} & -\frac{1}{2} \\
\frac{1}{2} s_{y} & \frac{\sqrt{N^{2}-4}}{4} s_{y} & -\frac{1}{2} & -\frac{N}{4}
\end{array}\right) \frac{1}{2} \lambda
\end{aligned}
$$

where $s_{y}=\operatorname{sgn}(y)$ and, like $\rho, \lambda$ is small in the region of interest (it is defined in [7]). The only property that we shall need here is that, in the final-state collinear limit, $\lambda=$ $\rho(Y, 2|y|)=\rho(Y, \Delta y)$.

We are now ready to calculate $\sigma_{1}$. The all-orders calculation was discussed in [7]. Here we focus on its order-by-order expansion in the initial-state collinear limit. Specifically we consider the out-of-gap gluon to be collinear to the incoming quark with momentum $p_{1}$, i.e. $y \rightarrow \infty$ at fixed $k_{T}$. Here $\gamma$ and $\mathbf{D}^{\mu}$ simplify, since $h_{1} \gg h_{2} \sim h_{3} \sim h_{4} \equiv h$, implying corresponding results for $\omega_{i j}$. We obtain two contributions, which it will be useful to keep separate in anticipation of our comparison with the result obtained using the basis independent method. The first contribution is when the out-of-gap gluon (either real or virtual) is hardest. By that we mean that is has the largest $k_{T}$ of all the soft gluons that dress the primary hard scatter. It is accompanied by two Coulomb gluons and an eikonal 
gluon, all at lower $k_{T}$. Replacing the $y$ integration by $2 \ln \left(Q / k_{T}\right)$, as explained in [7], and integrating over $k_{T}$ we obtain

$$
\sigma_{1, \text { out }=\text { hardest }}=-\sigma_{0}\left(\frac{2 \alpha_{s}}{\pi}\right)^{4} \ln ^{5}\left(\frac{Q}{Q_{0}}\right) \pi^{2} Y \frac{N^{2}-2}{240} .
$$

The second contribution comes when the out-of-gap gluon is the second hardest. It is accompanied by one harder Coulomb gluon and two lower $k_{T}$ gluons (one eikonal and one Coulomb). In this case we obtain

$$
\sigma_{1, \text { out }=\text { second-hardest }}=-\sigma_{0}\left(\frac{2 \alpha_{s}}{\pi}\right)^{4} \ln ^{5}\left(\frac{Q}{Q_{0}}\right) \pi^{2} Y \frac{N^{2}-1}{120}
$$

Summing the two contributions, we obtain the result in equation (3.24) of [7] (up to a factor of two, since the result there is for an out-of-gap gluon with $y>0$, while the factor of 2 introduced in the $y$ integration above accounts for the fact that the out-of-gap gluon could be on either side of the gap):

$$
\sigma_{1}=-\sigma_{0}\left(\frac{2 \alpha_{s}}{\pi}\right)^{4} \ln ^{5}\left(\frac{Q}{Q_{0}}\right) \pi^{2} Y \frac{3 N^{2}-4}{240} .
$$

We will use these results in section 4, as a cross-check of the basis independent results.

\section{Colour basis independent notation}

The colour basis independent notation we use was developed by Catani, Marchesini and others (for examples, see [16, 20-23]). In this section we introduce it before using it to derive the anomalous dimension matrix for a rapidity gap in an arbitrary ( $m$-parton) final state.

We start by considering the amplitude for the emission of a soft gluon off an $m$-parton amplitude. We can write

$$
|m+1\rangle=g \sum_{i} \frac{p_{i} \cdot \epsilon^{*}}{p_{i} \cdot k} \mathbf{T}_{i}^{a}|m\rangle
$$

where $g$ is the strong coupling constant, $k$ is the momentum of the emitted gluon and $\epsilon$ is its polarization vector. The ket $|m\rangle$ represents the amplitude prior to emission and makes explicit that it is a vector in colour space. The space is spanned by a set of basis kets $|i\rangle$ which we can take to form an orthonormal set. $\mathbf{T}_{i}^{a}$ is the operator that determines the map from the $m$ dimensional vector space onto the $m+1$ dimensional space which occurs as a result of emitting a gluon of colour $a$. One might choose to represent the $m$-parton amplitude by $M_{i_{1} i_{2} i_{3} \cdots i_{m}}$ where the indices are the colour indices of incoming or outgoing quarks, antiquarks or gluons. In such a representation, the $\mathbf{T}_{i}^{a}$ in eq. (3.1) will be represented by $\mathbf{t}^{a}$, the generator in the fundamental representation, if parton $i$ is either an outgoing quark or an incoming antiquark. The sign reverses if $i$ is an incoming quark or an outgoing antiquark. Similarly, if the radiating parton is a gluon we should use the generator in the adjoint representation, $-i \mathbf{f}^{a}$. In all cases, it is to be understood that eq. (3.1) provides the definition of the sign convention of the soft gluon emission vertex. The 
Hermitian conjugate operator $\left(\mathbf{T}_{i}^{a}\right)^{\dagger}$ determines the map from the $m+1$ dimensional vector space to the $m$ dimensional space corresponding to the absorption of a gluon of colour $a$.

Under a general $\mathrm{SU}(3)$ transformation, $|m\rangle$ transforms as a colour singlet and since the generators in the $m$-parton representation correspond to $\sum_{i=1}^{m} \mathbf{T}_{i}^{a}$ it follows that

$$
\sum_{i=1}^{m} \mathbf{T}_{i}^{a}|m\rangle=0 .
$$

This identity will prove to be very useful. Also of note is the fact that

$$
\left(\mathbf{T}_{i}^{a}\right)^{\dagger} \mathbf{T}_{j}^{a}=\left(\mathbf{T}_{j}^{a}\right)^{\dagger} \mathbf{T}_{i}^{a}
$$

as a result of which we introduce the notation

$$
\left(\mathbf{T}_{i}^{a}\right)^{\dagger} \mathbf{T}_{j}^{a} \equiv \mathbf{T}_{i} \cdot \mathbf{T}_{j}
$$

In this basis independent framework, we can write down the anomalous dimension matrix for an arbitrary phase-space veto in an arbitrary $m$-parton process. It is

$$
\boldsymbol{\Gamma}=-\sum_{i<j} \mathbf{T}_{i} \cdot \mathbf{T}_{j} \Omega_{i j}
$$

where

$$
\Omega_{i j}=\frac{1}{2}\left\{\int_{\text {veto }} \frac{d y d \phi}{2 \pi} \omega_{i j}-i \pi \Theta(i j=I I \text { or } F F)\right\},
$$

the sum over $i, j$ runs over all partons in the initial and final state and the ordering $i<j$ is simply to ensure that each pair is counted once, since $\mathbf{T}_{i} \cdot \mathbf{T}_{j}$ and $\Omega_{i j}$ are both symmetric under interchange of $i$ and $j .^{3}$ Note that, apart from the sign convention $\left(\Omega_{i j}\right.$ always takes a plus sign in this paper) this is the same definition as that used in [19]. The theta function ensures that the $i \pi$ contribution is present only when $i j$ correspond to a pair of incoming $(I I)$ or outgoing $(F F)$ partons.

$\Omega_{i j}$ was calculated in [19] for the case of an azimuthally-symmetric gap in rapidity of length $Y$, with sufficient generality for arbitrary $i, j$ kinematics. It can be summarized as

$$
\begin{aligned}
\Omega_{i j}=\frac{1}{2}\{ & Y \Theta(i j \text { on opposite sides of gap })+\frac{1}{2} \rho\left(Y ; 2\left|y_{i}\right|\right)+\frac{1}{2} \rho\left(Y ; 2\left|y_{j}\right|\right) \\
& \left.-\lambda\left(Y ;\left|y_{i}\right|+\left|y_{j}\right|,\left|\phi_{i}-\phi_{j}\right|\right) \Theta(i j \text { on same side of gap })-i \pi \Theta(i j=I I \text { or } F F)\right\},
\end{aligned}
$$

where $\rho$ and $\lambda$ are known functions, the only properties of which we will need here are:

1. $\rho(Y ;|y|), \lambda(Y ;|y|, \Delta \phi) \rightarrow 0$ as $|y| \rightarrow \infty$; and

2. $\lambda(Y ;|y|, \Delta \phi) \rightarrow \rho(Y ;|y|)$ as $\Delta \phi \rightarrow 0$.

\footnotetext{
${ }^{3}$ We work in Feynman gauge and assume massless partons, thereby avoiding self-energy contributions with $i=j$.
} 
The first property ensures that $\rho$ and $\lambda$ are absent for initial-state partons.

The simplicity of the result for $\Omega_{i j}$ allows one to simplify the result for $\boldsymbol{\Gamma}$. Gathering together terms with the same momentum dependence, we obtain

$$
\begin{aligned}
\boldsymbol{\Gamma}= & -\frac{1}{2} Y\left(\sum_{i \in L} \mathbf{T}_{i}\right) \cdot\left(\sum_{j \in R} \mathbf{T}_{j}\right)+\frac{1}{2} i \pi\left(\mathbf{T}_{1} \cdot \mathbf{T}_{2}+\sum_{(i<j) \in F} \mathbf{T}_{i} \cdot \mathbf{T}_{j}\right) \\
& -\frac{1}{4} \sum_{i \in F} \rho\left(Y ; 2\left|y_{i}\right|\right) \sum_{j \neq i} \mathbf{T}_{i} \cdot \mathbf{T}_{j} \\
& +\frac{1}{2} \sum_{(i<j) \in L} \lambda\left(Y ;\left|y_{i}\right|+\left|y_{j}\right|,\left|\phi_{i}-\phi_{j}\right|\right) \mathbf{T}_{i} \cdot \mathbf{T}_{j}+\frac{1}{2} \sum_{(i<j) \in R} \lambda\left(Y ;\left|y_{i}\right|+\left|y_{j}\right|,\left|\phi_{i}-\phi_{j}\right|\right) \mathbf{T}_{i} \cdot \mathbf{T}_{j},
\end{aligned}
$$

where the labels $L$ and $R$ label the bunches of partons on each side of the gap and the indices " 1 " and "2" refer to the two incoming partons. Now we can use colour conservation to simplify this expression further. Firstly we have

$$
\sum_{j \neq i} \mathbf{T}_{i} \cdot \mathbf{T}_{j}=-\mathbf{T}_{i}^{2}
$$

and secondly we can perform a similar trick on the Coulomb gluon terms:

$$
\sum_{(i<j) \in F} \mathbf{T}_{i} \cdot \mathbf{T}_{j}=\mathbf{T}_{1} \cdot \mathbf{T}_{2}+\frac{1}{2}\left(\sum_{i \in I} \mathbf{T}_{i}^{2}-\sum_{i \in F} \mathbf{T}_{i}^{2}\right) .
$$

Now, adding an imaginary multiple of the identity matrix to the anomalous dimension matrix has no physical effect, so we are free to drop the $\mathbf{T}_{i}^{2}$ terms. The Coulomb gluon terms are thus proportional to $\mathbf{T}_{1} \cdot \mathbf{T}_{2}$ and are absent if one or both of the incoming partons is colourless. This was first pointed out using the colour basis independent notation in 16. and is an important component of the proofs of factorization in 24-26.

Finally, we can re-write the leading $(\sim Y)$ eikonal gluon term using

$$
\left(\sum_{i \in L} \mathbf{T}_{i}\right) \cdot\left(\sum_{j \in R} \mathbf{T}_{j}\right)=-\left(\sum_{i \in L} \mathbf{T}_{i}\right)^{2}=-\left(\sum_{i \in R} \mathbf{T}_{i}\right)^{2} \equiv-\mathbf{T}_{t}^{2} .
$$

That is, if we think of the rapidity gap as separating the partonic event into two separate systems, the dominant Sudakov suppression effectively comes from emission off the total colour charge exchanged between the two systems, as noticed in [27].

Thus the full result for the anomalous dimension matrix for an azimuthally symmetric rapidity gap of length $Y$ is given by

$$
\begin{aligned}
\boldsymbol{\Gamma}= & \frac{1}{2} Y \mathbf{T}_{t}^{2}+i \pi \mathbf{T}_{1} \cdot \mathbf{T}_{2}+\frac{1}{4} \sum_{i \in F} \rho\left(Y ; 2\left|y_{i}\right|\right) \mathbf{T}_{i}^{2} \\
& +\frac{1}{2} \sum_{(i<j) \in L} \lambda\left(Y ;\left|y_{i}\right|+\left|y_{j}\right|,\left|\phi_{i}-\phi_{j}\right|\right) \mathbf{T}_{i} \cdot \mathbf{T}_{j}+\frac{1}{2} \sum_{(i<j) \in R} \lambda\left(Y ;\left|y_{i}\right|+\left|y_{j}\right|,\left|\phi_{i}-\phi_{j}\right|\right) \mathbf{T}_{i} \cdot \mathbf{T}_{j} .
\end{aligned}
$$



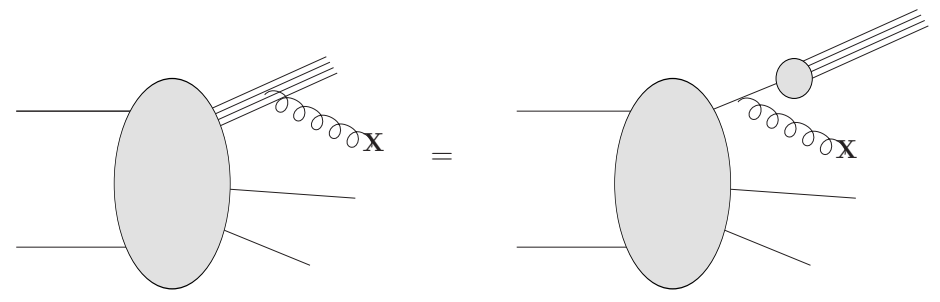

(a)
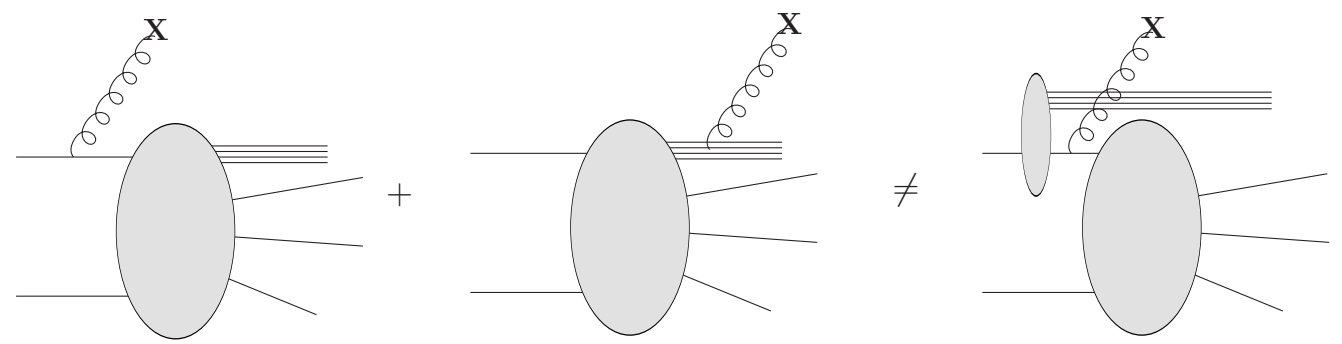

(b)

Figure 1: Factorization of soft gluon emission off a collinear bunch of partons. The cross indicates that the gluon can be attached to any other external leg. A sum over couplings to the final state collinear partons is implied.

Notice that the terms involving $\rho$ are Abelian in nature since $\mathbf{T}_{i}^{2}=C_{F} \mathbb{1}$ or $C_{A} \mathbb{1}$ depending upon whether parton $i$ is a quark/antiquark or gluon.

Now we can prove a very important property of $\boldsymbol{\Gamma}$ : it is safe against final state collinear singularities. More specifically, if any two or more partons in the final state become collinear with each other, the soft gluon evolution of the system is identical to the evolution of the system in which the collinear partons are replaced by a single parton with the same total colour charge. That is, if $k$ and $l$ are the collinear partons, then $\boldsymbol{\Gamma}$ depends only upon $\mathbf{T}_{k}+\mathbf{T}_{l}$ and not upon the $\mathbf{T}_{k}$ or $\mathbf{T}_{l}$ separately. The proof is straightforward. Let us consider partons $k$ and $l$ to be final state and collinear. ${ }^{4}$ We first note that since the imaginary part of $\boldsymbol{\Gamma}$ can be written in terms of $\mathbf{T}_{1} \cdot \mathbf{T}_{2}$ only, it has no explicit dependence on $\mathbf{T}_{k}$ and $\mathbf{T}_{l}$ and we need only consider the real part of $\boldsymbol{\Gamma}$. The part of $\boldsymbol{\Gamma}$ that depends upon the colour charges of $k$ and $l$ is

$$
\mathbb{R e}\left(\boldsymbol{\Gamma}^{(k l)}\right)=\sum_{i \neq k, l} \mathbf{T}_{i} \cdot \mathbf{T}_{k} \mathbb{R e}\left(\Omega_{i k}\right)+\sum_{i \neq k, l} \mathbf{T}_{i} \cdot \mathbf{T}_{l} \mathbb{R e}\left(\Omega_{i l}\right)+\mathbf{T}_{k} \cdot \mathbf{T}_{l} \operatorname{Re}\left(\Omega_{k l}\right) .
$$

Now since $k$ and $l$ are collinear $\mathbb{R e}\left(\Omega_{i k}\right)=\mathbb{R e}\left(\Omega_{i l}\right)$. The equality follows since $\omega_{i j}$ depends only on the direction of partons $i$ and $j$ and not their energies. Moreover, $\operatorname{Re}\left(\Omega_{k l}\right)$ vanishes in the collinear limit since the numerator of $\omega_{k l}$ vanishes. It now follows immediately that $\boldsymbol{\Gamma}^{(k l)}$ depends only upon the sum $\mathbf{T}_{k}+\mathbf{T}_{l}$ and hence that soft gluons factorize from collinear final state emissions as illustrated in figure 1(a).

We contrast this result with that in the initial state collinear limit, in which one or more outgoing partons becomes collinear with one of the incoming partons. Precisely the

\footnotetext{
${ }^{4}$ The generalization to more than two collinear partons is straightforward.
} 
same reasoning as before can be used for the real part of $\boldsymbol{\Gamma}$, but since the imaginary part can be written in terms of the colours of the initial state partons only, it does not depend on the sum of the colour charges of the collinear partons and the factorization is broken, as illustrated in figure 1 (b).

It is this fact that we described in [11, 12, as a breakdown of naive coherence. It leads directly to the appearance of super-leading logarithms in the calculation of the gapsbetween-jets cross-section.

\section{One gluon outside the gap}

We now proceed to calculate the one gluon outside the gap cross-section according to eqs. (2.8)-(2.10) in the colour basis independent notation. Since we require the colour evolution of the $m$ and $m+1$ parton systems, we modify the notation slightly, to differentiate between the colour matrix to emit a gluon from a parton $i$ in the $m$ parton system, $\mathbf{t}_{i}$, and in the $m+1$ parton system, $\mathbf{T}_{i}$. We continue to use the notation from [0] and [19] in which the additional gluon in the $m+1$-parton system is labelled $k$, whilst the others are labelled by indices running over the range 1 to $m$.

Without loss of generality, we assume that the gluon is emitted on the same side of the gap as partons 1 and 3 and, from eq. (3.12), it follows that

$$
\begin{aligned}
\boldsymbol{\Gamma}= & \frac{1}{2} Y \mathbf{t}_{t}^{2}+i \pi \mathbf{t}_{1} \cdot \mathbf{t}_{2}+\frac{1}{4} \rho(Y ; \Delta y)\left(\mathbf{t}_{3}^{2}+\mathbf{t}_{4}^{2}\right), \\
\boldsymbol{\Lambda}= & \frac{1}{2} Y \mathbf{T}_{t}^{2}+i \pi \mathbf{T}_{1} \cdot \mathbf{T}_{2}+\frac{1}{4} \rho(Y ; \Delta y)\left(\mathbf{T}_{3}^{2}+\mathbf{T}_{4}^{2}\right)+\frac{1}{4} \rho(Y ; 2 y) \mathbf{T}_{k}^{2} \\
& +\frac{1}{2} \lambda\left(Y ; \frac{1}{2} \Delta y+y, \phi\right) \mathbf{T}_{3} \cdot \mathbf{T}_{k},
\end{aligned}
$$

with $\mathbf{t}_{t}^{2}=\left(\mathbf{t}_{1}+\mathbf{t}_{3}\right)^{2}=\left(\mathbf{t}_{2}+\mathbf{t}_{4}\right)^{2}$ and $\mathbf{T}_{t}^{2}=\left(\mathbf{T}_{1}+\mathbf{T}_{3}+\mathbf{T}_{k}\right)^{2}=\left(\mathbf{T}_{2}+\mathbf{T}_{4}\right)^{2}$. We also require the real and virtual emission matrices, which are given by

$$
\begin{aligned}
\mathbf{D}_{a}^{\mu} & =\sum_{i} \mathbf{t}_{i}^{a} h_{i}^{\mu}, \\
\gamma & =-\frac{1}{2} \sum_{i<j} \mathbf{t}_{i} \cdot \mathbf{t}_{j} \omega_{i j} .
\end{aligned}
$$

Now we specialize to the case where the gluon is collinear with incoming parton 1 , i.e. it has $y \rightarrow \infty$. In this case, the last two terms of $\boldsymbol{\Lambda}$ vanish. The remaining $\rho$ terms are once again Abelian, and the same for both final states, so they lead only to an overall factor, which we neglect in the following discussion. The real and virtual emission matrices also simplify in this limit, and we have

$$
\begin{aligned}
\boldsymbol{\Gamma} & =\frac{1}{2} Y \mathbf{t}_{t}^{2}+i \pi \mathbf{t}_{1} \cdot \mathbf{t}_{2}, \\
\boldsymbol{\Lambda} & =\frac{1}{2} Y \mathbf{T}_{t}^{2}+i \pi \mathbf{T}_{1} \cdot \mathbf{T}_{2}, \\
\mathbf{D}_{a}^{\mu} & =\left(h_{1}^{\mu}-h^{\mu}\right) \mathbf{t}_{1}^{a}, \\
\boldsymbol{\gamma} & =\frac{1}{2} \mathbf{t}_{1}^{2} .
\end{aligned}
$$


In eq. (4.7) we have introduced $h^{\mu}$ to emphasize the fact that in the collinear limit all the $h_{i}^{\mu}$ for $i \neq 1$ are equal. Note that we have used $\left(h_{1}-h\right) \cdot\left(h_{1}-h\right)=-1$ to simplify $\gamma$. The cross-section can then be written as

$$
\begin{gathered}
\sigma_{1}=-\frac{2 \alpha_{s}}{\pi} \int_{Q_{0}}^{Q} \frac{d k_{T}}{k_{T}}\left(2 \ln \frac{Q}{k_{T}}\right)\left\langle m_{0}\right| e^{-\frac{2 \alpha_{s}}{\pi} \int_{k_{T}}^{Q} \frac{d k_{T}^{\prime}}{k_{T}^{\prime}}\left(\frac{1}{2} Y \mathbf{t}_{t}^{2}-i \pi \mathbf{t}_{1} \cdot \mathbf{t}_{2}\right)} \\
\left\{\mathbf{t}_{1}^{2} e^{-\frac{2 \alpha_{s}}{\pi} \int_{Q_{0}}^{k_{T}} \frac{d k_{T}^{\prime}}{k_{T}^{\prime}}\left(\frac{1}{2} Y \mathbf{t}_{t}^{2}-i \pi \mathbf{t}_{1} \cdot \mathbf{t}_{2}\right)} e^{-\frac{2 \alpha_{s}}{\pi} \int_{Q_{0}}^{k_{T}} \frac{d k_{T}^{\prime}}{k_{T}^{\prime}}\left(\frac{1}{2} Y \mathbf{t}_{t}^{2}+i \pi \mathbf{t}_{1} \cdot \mathbf{t}_{2}\right)}\right. \\
\left.-\mathbf{t}_{1}^{a \dagger} e^{-\frac{2 \alpha_{s}}{\pi} \int_{Q_{0}}^{k_{T}} \frac{d k_{T}^{\prime}}{k_{T}^{\prime}}\left(\frac{1}{2} Y \mathbf{T}_{t}^{2}-i \pi \mathbf{T}_{1} \cdot \mathbf{T}_{2}\right)} e^{-\frac{2 \alpha_{s}}{\pi} \int_{Q_{0}}^{k_{T}} \frac{d k_{T}^{\prime}}{k_{T}^{\prime}}\left(\frac{1}{2} Y \mathbf{T}_{t}^{2}+i \pi \mathbf{T}_{1} \cdot \mathbf{T}_{2}\right)} \mathbf{t}_{1}^{a}\right\} \\
e^{-\frac{2 \alpha_{s}}{\pi} \int_{k_{T}}^{Q} \frac{d k_{T}^{\prime}}{k_{T}^{\prime}}\left(\frac{1}{2} Y \mathbf{t}_{t}^{2}+i \pi \mathbf{t}_{1} \cdot \mathbf{t}_{2}\right)}\left|m_{0}\right\rangle .
\end{gathered}
$$

Note that it is the non-commutativity of $\mathbf{T}_{t}^{2}$ and $\mathbf{T}_{1} \cdot \mathbf{T}_{2}$ (and similarly $\mathbf{t}_{t}^{2}$ and $\mathbf{t}_{1} \cdot \mathbf{t}_{2}$ ) that prevents this expression from cancelling to zero: if they commuted then the two exponentials could be combined, all $\mathbf{T}_{1} \cdot \mathbf{T}_{2}$ and $\mathbf{t}_{1} \cdot \mathbf{t}_{2}$ dependence would cancel, $\mathbf{t}_{1}$ could be commuted through $\mathbf{T}_{t}^{2}$ and the real and virtual parts would be identical.

To find the first non-zero super-leading logarithm, we expand the main bracket of this expression order by order in $\alpha_{s}$ :

$$
\begin{aligned}
& \left\{\begin{array}{l}
\}_{0}=\mathbf{t}_{1}^{2}-\mathbf{t}_{1}^{a \dagger} \mathbf{t}_{1}^{a}=0 . \\
\{\}_{1}=-\frac{2 \alpha_{s}}{\pi} \int_{Q_{0}}^{k_{T}} \frac{d k_{T}^{\prime}}{k_{T}^{\prime}}\left\{\mathbf{t}_{1}^{2} Y \mathbf{t}_{t}^{2}-\mathbf{t}_{1}^{a \dagger} Y \mathbf{T}_{t}^{2} \mathbf{t}_{1}^{a}\right\}
\end{array}\right.
\end{aligned}
$$

is also zero because

$$
\mathbf{T}_{t}^{2} \mathbf{t}_{1}^{a}=\mathbf{t}_{1}^{a} \mathbf{t}_{t}^{2}
$$

Expanding to order $\alpha_{s}^{2}$ yields

$$
\{\}_{2}=\left(\frac{i \pi Y}{2}\right)\left(-\frac{2 \alpha_{s}}{\pi} \int_{Q_{0}}^{k_{T}} \frac{d k_{T}^{\prime}}{k_{T}^{\prime}}\right)^{2}\left\{\mathbf{t}_{1}^{2}\left[\mathbf{t}_{t}^{2}, \mathbf{t}_{1} \cdot \mathbf{t}_{2}\right]-\mathbf{t}_{1}^{a \dagger}\left[\mathbf{T}_{t}^{2}, \mathbf{T}_{1} \cdot \mathbf{T}_{2}\right] \mathbf{t}_{1}^{a}\right\} .
$$

Note that this result comes only from the case where there is one Coulomb gluon and one eikonal gluon either side of the cut. Now, this term is not zero but the corresponding matrix element is zero, i.e.

$$
\left\langle m_{0}\left|\{\}_{2}\right| m_{0}\right\rangle=0 .
$$

This term will however be relevant at the next order (when we add an additional Coulomb gluon) and so we take the opportunity here to simplify it further. Using colour conservation, and the fact that $\mathbf{t}_{1} \cdot \mathbf{t}_{2}$ commutes with itself, with all colour dot products involving only final state particles and with all $\mathbf{t}_{i}^{2}$, we obtain

$$
\left[\mathbf{t}_{t}^{2}, \mathbf{t}_{1} \cdot \mathbf{t}_{2}\right]=-2\left[\mathbf{t}_{1} \cdot \mathbf{t}_{4}, \mathbf{t}_{1} \cdot \mathbf{t}_{2}\right] \quad \text { and } \quad\left[\mathbf{T}_{t}^{2}, \mathbf{T}_{1} \cdot \mathbf{T}_{2}\right]=-2\left[\mathbf{T}_{1} \cdot \mathbf{T}_{4}, \mathbf{T}_{1} \cdot \mathbf{T}_{2}\right]
$$


Thus we can simplify eq. (4.13) by replacing the commutator $\left[\mathbf{t}_{t}^{2}, \mathbf{t}_{1} \cdot \mathbf{t}_{2}\right]$ with $-2\left[\mathbf{t}_{1} \cdot \mathbf{t}_{4}, \mathbf{t}_{1} \cdot \mathbf{t}_{2}\right]$, and similarly for $\left[\mathbf{T}_{t}^{2}, \mathbf{T}_{1} \cdot \mathbf{T}_{2}\right]$.

At the next order we obtain

$$
\begin{array}{r}
\{\}_{3}=\frac{1}{3 !}\left(-\frac{2 \alpha_{s}}{\pi} \int_{Q_{0}}^{k_{T}} \frac{d k_{T}^{\prime}}{k_{T}^{\prime}}\right)^{3}\left\{\mathbf { t } _ { 1 } ^ { 2 } \left(\left(Y \mathbf{t}_{t}^{2}\right)^{3}+\frac{3}{2} Y^{2} i \pi\left(\left(\mathbf{t}_{t}^{2}\right)^{2} \mathbf{t}_{1} \cdot \mathbf{t}_{2}-\mathbf{t}_{1} \cdot \mathbf{t}_{2}\left(\mathbf{t}_{t}^{2}\right)^{2}\right)\right.\right. \\
\left.-Y \pi^{2}\left(\mathbf{t}_{t}^{2} \mathbf{t}_{1} \cdot \mathbf{t}_{2} \mathbf{t}_{1} \cdot \mathbf{t}_{2}-2 \mathbf{t}_{1} \cdot \mathbf{t}_{2} \mathbf{t}_{t}^{2} \mathbf{t}_{1} \cdot \mathbf{t}_{2}+\mathbf{t}_{1} \cdot \mathbf{t}_{2} \mathbf{t}_{1} \cdot \mathbf{t}_{2} \mathbf{t}_{t}^{2}\right)\right) \\
-\mathbf{t}_{1}^{a \dagger}\left(\left(Y \mathbf{T}_{t}^{2}\right)^{3}+\frac{3}{2} Y^{2} i \pi\left(\left(\mathbf{T}_{t}^{2}\right)^{2} \mathbf{T}_{1} \cdot \mathbf{T}_{2}-\mathbf{T}_{1} \cdot \mathbf{T}_{2}\left(\mathbf{T}_{t}^{2}\right)^{2}\right)\right. \\
\left.\left.-Y \pi^{2}\left(\mathbf{T}_{t}^{2} \mathbf{T}_{1} \cdot \mathbf{T}_{2} \mathbf{T}_{1} \cdot \mathbf{T}_{2}-2 \mathbf{T}_{1} \cdot \mathbf{T}_{2} \mathbf{T}_{t}^{2} \mathbf{T}_{1} \cdot \mathbf{T}_{2}+\mathbf{T}_{1} \cdot \mathbf{T}_{2} \mathbf{T}_{1} \cdot \mathbf{T}_{2} \mathbf{T}_{t}^{2}\right)\right) \mathbf{t}_{1}^{a}\right\}
\end{array}
$$

Note that the 3 ! seems to imply that only contributions where all three gluons are on the same side of the cut are relevant. This is not the case: the $2 Y \pi^{2} \mathbf{t}_{1} \cdot \mathbf{t}_{2} \mathbf{t}_{t}^{2} \mathbf{t}_{1} \cdot \mathbf{t}_{2}$ term actually also has a contribution from when the two Coulomb gluons lie on opposite sides of the cut, i.e. $2 / 3 !=1 / 2$ ! $-1 / 3$ !. Of the various terms, the first term $\left(\sim Y^{3}\right)$ cancels between the real and virtual contributions and the second $\left(\sim Y^{2} \pi\right.$ term) vanishes upon forming the matrix element. We are thus left with a leading contribution only from the third $\left(\sim Y \pi^{2}\right)$ term:

$$
\begin{aligned}
\{\}_{3} \equiv-\frac{Y \pi^{2}}{6}\left(-\frac{2 \alpha_{s}}{\pi} \int_{Q_{0}}^{k_{T}} \frac{d k_{T}^{\prime}}{k_{T}^{\prime}}\right)^{3}\{ & \mathbf{t}_{1}^{2}\left[\left[\mathbf{t}_{t}^{2}, \mathbf{t}_{1} \cdot \mathbf{t}_{2}\right], \mathbf{t}_{1} \cdot \mathbf{t}_{2}\right] \\
& \left.-\mathbf{t}_{1}^{a \dagger}\left[\left[\mathbf{T}_{t}^{2}, \mathbf{T}_{1} \cdot \mathbf{T}_{2}\right], \mathbf{T}_{1} \cdot \mathbf{T}_{2}\right] \mathbf{t}_{1}^{a}\right\}
\end{aligned}
$$

As before, $\mathbf{t}_{t}^{2}$ can be replaced by $-2 \mathbf{t}_{1} \cdot \mathbf{t}_{4}$ (and similarly for $\mathbf{T}_{t}^{2}$ ).

Substituting back into eq. (4.9), we obtain a contribution to the first super-leading logarithm from configurations in which the out-of-gap gluon is hardest ${ }^{5}$ of

$$
\begin{aligned}
& \sigma_{1, \text { out }=\text { hardest }}=\left(\frac{2 \alpha_{s}}{\pi}\right)^{4} \int_{Q_{0}}^{Q} \frac{d k_{T}}{k_{T}}\left(2 \ln \frac{Q}{k_{T}}\right)\left(\int_{Q_{0}}^{k_{T}} \frac{d k_{T}^{\prime}}{k_{T}^{\prime}}\right)^{3} \frac{Y \pi^{2}}{3} \\
& \quad \times\left\langle m_{0}\left|\mathbf{t}_{1}^{2}\left[\left[\mathbf{t}_{1} \cdot \mathbf{t}_{4}, \mathbf{t}_{1} \cdot \mathbf{t}_{2}\right], \mathbf{t}_{1} \cdot \mathbf{t}_{2}\right]-\mathbf{t}_{1}^{a \dagger}\left[\left[\mathbf{T}_{1} \cdot \mathbf{T}_{4}, \mathbf{T}_{1} \cdot \mathbf{T}_{2}\right], \mathbf{T}_{1} \cdot \mathbf{T}_{2}\right] \mathbf{t}_{1}^{a}\right| m_{0}\right\rangle .
\end{aligned}
$$

The colour matrix element can be evaluated explicitly and after performing the transverse momentum integrals one finds the expression for $\sigma_{1, \text { out }=\text { hardest }}$ presented in eq. (2.20).

It is instructive to re-write eq. (4.18) in such a way as to make direct contact with the

\footnotetext{
${ }^{5}$ Obtained by setting the exponentials that lie outside of the main bracket in eq. (4.9) to unity.
} 
corresponding Feynman diagrams:

$$
\begin{aligned}
\sigma_{1, \text { out }=\text { hardest }}=2^{5}\left(-\frac{2 \alpha_{s}}{\pi} \int_{Q_{0}}^{Q} \frac{d k_{T}}{k_{T}}\right)\left(\ln \frac{Q}{k_{T}}\right)\left(-\frac{2 \alpha_{s}}{\pi} \int_{Q_{0}}^{k_{T}} \frac{d k_{T}^{\prime}}{k_{T}^{\prime}}\right)^{3} \\
\times\left\langle m_{0}\right|\left[\frac{1}{3 !}\left(-\frac{1}{2} Y \mathbf{t}_{1} \cdot \mathbf{t}_{4}\right)\left(+\frac{1}{2} i \pi \mathbf{t}_{1} \cdot \mathbf{t}_{2}\right)\left(+\frac{1}{2} i \pi \mathbf{t}_{1} \cdot \mathbf{t}_{2}\right)\right. \\
+\frac{1}{3 !}\left(+\frac{1}{2} i \pi \mathbf{t}_{1} \cdot \mathbf{t}_{2}\right)\left(+\frac{1}{2} i \pi \mathbf{t}_{1} \cdot \mathbf{t}_{2}\right)\left(-\frac{1}{2} Y \mathbf{t}_{1} \cdot \mathbf{t}_{4}\right) \\
+\frac{1}{2 !}\left(-\frac{1}{2} i \pi \mathbf{t}_{1} \cdot \mathbf{t}_{2}\right)\left(-\frac{1}{2} Y \mathbf{t}_{1} \cdot \mathbf{t}_{4}\right)\left(+\frac{1}{2} i \pi \mathbf{t}_{1} \cdot \mathbf{t}_{2}\right) \\
\left.+\frac{1}{3 !}\left(+\frac{1}{2} i \pi \mathbf{t}_{1} \cdot \mathbf{t}_{2}\right)\left(-\frac{1}{2} Y \mathbf{t}_{1} \cdot \mathbf{t}_{4}\right)\left(+\frac{1}{2} i \pi \mathbf{t}_{1} \cdot \mathbf{t}_{2}\right)\right] 2\left(\frac{1}{2} \mathbf{t}_{1}^{2}\right) \\
-2\left(\frac{1}{2} \mathbf{t}_{1}^{a \dagger}\right)\left[\frac{1}{3 !}\left(-\frac{1}{2} Y \mathbf{T}_{1} \cdot \mathbf{t}_{4}\right)\left(+\frac{1}{2} i \pi \mathbf{T}_{1} \cdot \mathbf{T}_{2}\right)\left(+\frac{1}{2} i \pi \mathbf{T}_{1} \cdot \mathbf{T}_{2}\right)\right. \\
+\frac{1}{3 !}\left(+\frac{1}{2} i \pi \mathbf{T}_{1} \cdot \mathbf{T}_{2}\right)\left(+\frac{1}{2} i \pi \mathbf{T}_{1} \cdot \mathbf{T}_{2}\right)\left(-\frac{1}{2} Y \mathbf{T}_{1} \cdot \mathbf{T}_{4}\right) \\
+\frac{1}{2 !}\left(-\frac{1}{2} i \pi \mathbf{T}_{1} \cdot \mathbf{T}_{2}\right)\left(-\frac{1}{2} Y \mathbf{T}_{1} \cdot \mathbf{T}_{4}\right)\left(+\frac{1}{2} i \pi \mathbf{T}_{1} \cdot \mathbf{T}_{2}\right) \\
\left.+\frac{1}{3 !}\left(+\frac{1}{2} i \pi \mathbf{T}_{1} \cdot \mathbf{T}_{2}\right)\left(-\frac{1}{2} Y \mathbf{T}_{1} \cdot \mathbf{T}_{4}\right)\left(+\frac{1}{2} i \pi \mathbf{T}_{1} \cdot \mathbf{T}_{2}\right)\right] \mathbf{t}_{1}^{a}\left|m_{0}\right\rangle .
\end{aligned}
$$

The eight terms in the matrix element correspond, in Feynman gauge, to the eight diagrams in figure 2, in the order (a), (g), (e), (c), (b), (h), (f), (d). The super-leading contribution arises when the soft gluons linking partons 1 and 2 are Coulomb gluons and the soft gluon shown linking partons 1 and 4 is an eikonal gluon. The hard gluons responsible for producing the hard scatter are represented by traditional curly lines. All the factors in this equation are understandable. The signs on $\alpha_{s}$ and all the $\mathbf{t}_{i} \cdot \mathbf{t}_{j}$ terms are the natural ones defined by our notation. ${ }^{6}$ Of the numerical factors, those on $\alpha_{s}$ and the $\mathbf{t}_{i} \cdot \mathbf{t}_{j}$ terms are again defined by our notation. The 3 ! arises when all three gluons are on the same side of the cut and accounts for the strong ordering in transverse momentum. Similarly the 2 ! in the middle term arises when two of the three gluons are on the same side of the cut. Of the five powers of two, two come from the fact that $i \pi \mathbf{t}_{1} \cdot \mathbf{t}_{2}$ is shorthand for the sum of all Coulomb diagrams, one comes from the fact that $-Y \mathbf{t}_{1} \cdot \mathbf{t}_{4}$ is shorthand for the sum over all gluons exchanged across the gap, one comes from accounting for the hermitian conjugate amplitudes and the final factor comes from allowing the out-of-gap gluon to be collinear to either of the incoming quarks. ${ }^{7}$ The $\frac{1}{2} \mathbf{t}_{1}^{2}$ term (and the corresponding $\frac{1}{2} \mathbf{t}_{1}^{a \dagger} \cdots \mathbf{t}_{1}^{a}$ term in the real emission case) deserves a little discussion. It is the colour factor corresponding to the out-of-gap gluon and as such it follows (in the collinear limit) after summing over

\footnotetext{
${ }^{6}$ Here and in the remainder of this paragraph we do not distinguish between $\mathbf{T}_{i}$ and $\mathbf{t}_{i}$.

${ }^{7}$ Strictly speaking this should be performed by summing over exchange of the identities of partons 1 and 2 when they are different, but since it turns out that the final result is symmetric in partons 1 and 2 , simply multiplying by 2 suffices.
} 


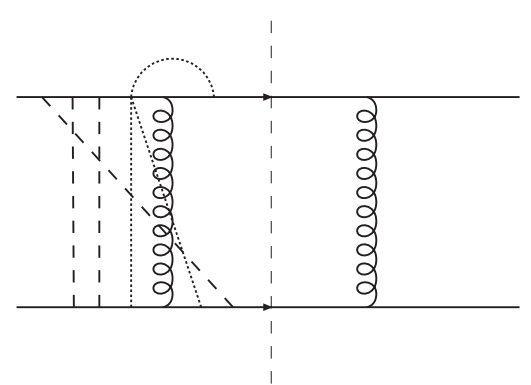

(a)

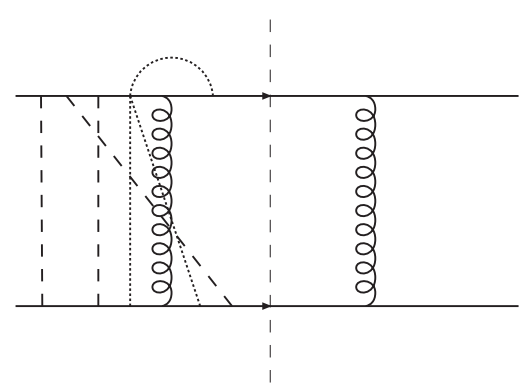

(c)

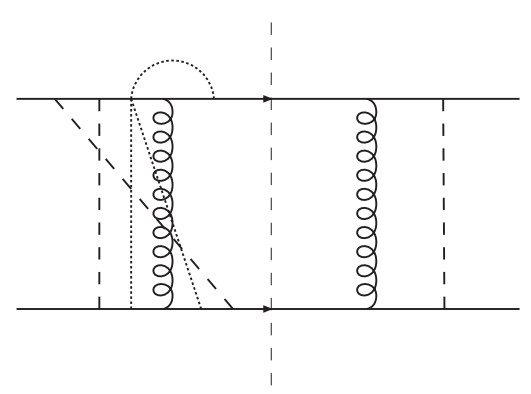

(e)

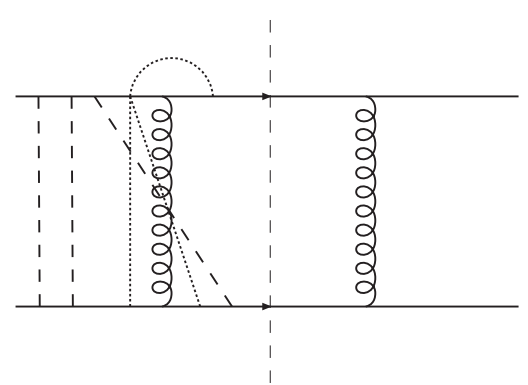

(g)

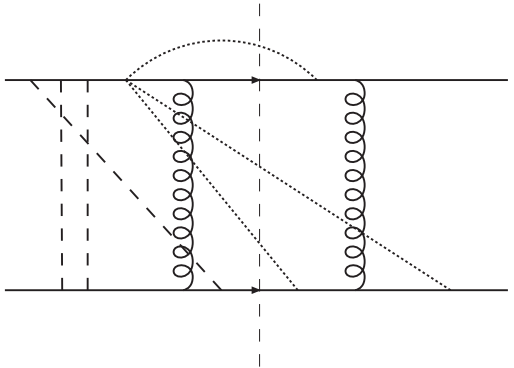

(b)

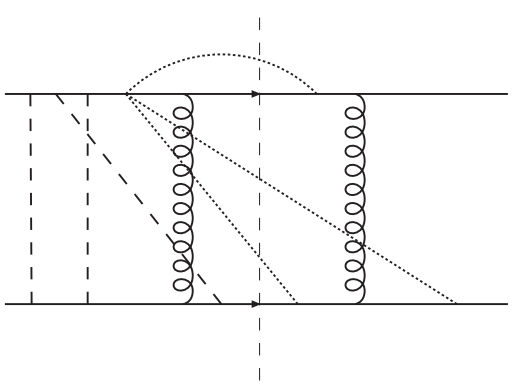

(d)

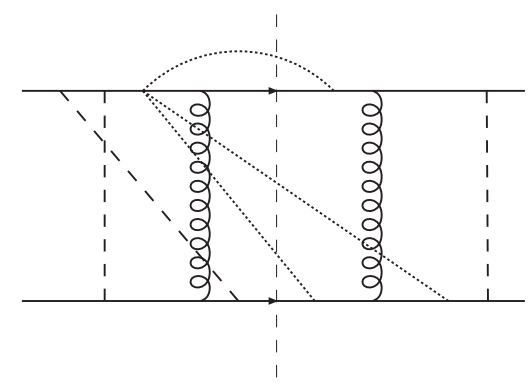

(f)

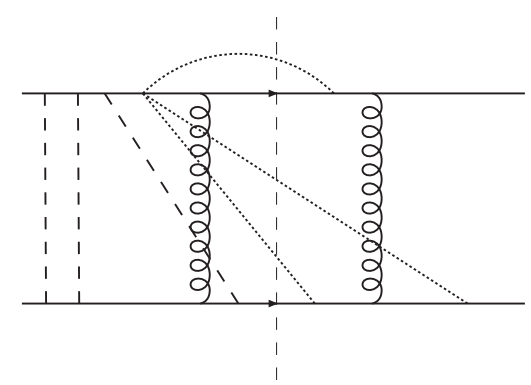

(h)

Figure 2: The relevant Feynman diagrams in the case that the out-of-gap (dotted) gluon is the hardest gluon. The dashed lines indicate soft (eikonal and Coulomb) gluons. Each subfigure represents three Feynman diagrams, corresponding to the three different ways of attaching the outof-gap gluon. In diagrams (e) and (f) the soft gluon to the right of the cut should only be integrated over the region in which it has transverse momentum less than the out-of-gap gluon. 
graphs where a gluon connects parton 1 with partons 2, 3 or 4 . Each of these attachments is illustrated by a different dotted line in the figures. The sum over diagrams leads to a colour diagonal contribution (a result that is more transparent in a physical gauge). The extra factor of 2 in front of the $\frac{1}{2} \mathbf{t}_{1}^{2}$ term comes from the fact that the virtual, out-of-gap, gluon can be either side of the cut, i.e. in figure 4.18 the $\mathbf{t}_{1}^{2}$ gluon could also appear to the right of the cut in each diagram. Similarly the real gluon could also be attached to parton 1 on the right of the cut and any other parton on the left of the cut. Strictly speaking this doubles the total number of graphs; although we do not show the additional $3 \times 8$ graphs (because they produce identical results in the collinear limit) they are distinct from the $3 \times 8$ shown in figure 2 .

To complete the calculation, we need to compute the contribution arising from the case where there is one virtual emission of higher $k_{T}$ than the out-of-gap emission. Now we use the expression for \{\}$_{2}$ derived in eq. (4.13) in conjunction with the order $\alpha_{s}$ expansion of the exponential factors that lie outside of the main bracket in eq. (4.9). Note that this is the only remaining contribution to the lowest order super-leading logarithm since all lower order expansions of the main bracket in eq. (4.9) (i.e. \{\}$_{1}$ and \{\}$_{0}$ ) vanish identically. The result is

$$
\begin{aligned}
\sigma_{1, \text { out }=\text { second-hardest }} & =\left(-\frac{2 \alpha_{s}}{\pi}\right)^{4} \int_{Q_{0}}^{Q} \frac{d k_{T}}{k_{T}}\left(2 \ln \frac{Q}{k_{T}}\right)\left(\int_{k_{T}}^{Q} \frac{d k_{T}^{\prime}}{k_{T}^{\prime}}\right)\left(\int_{Q_{0}}^{k_{T}} \frac{d k_{T}^{\prime \prime}}{k_{T}^{\prime \prime}}\right)^{2} \\
\times & \frac{i \pi Y}{2}(4 \pi i)\left\langle m_{0}\left|\left(\mathbf{t}_{1} \cdot \mathbf{t}_{2}\right)\left(\mathbf{t}_{1}^{2}\left[\mathbf{t}_{1} \cdot \mathbf{t}_{4}, \mathbf{t}_{1} \cdot \mathbf{t}_{2}\right]-\mathbf{t}_{1}^{a \dagger}\left[\mathbf{T}_{1} \cdot \mathbf{T}_{4}, \mathbf{T}_{1} \cdot \mathbf{T}_{2}\right] \mathbf{t}_{1}^{a}\right)\right| m_{0}\right\rangle .
\end{aligned}
$$

The factor $4 \pi i$ contains a factor of 2 for the contribution where the hardest Coulomb gluon is on the left of the cut, i.e. the $\left(\mathbf{t}_{1} \cdot \mathbf{t}_{2}\right)$ factor on the far left of the matrix element is moved to the far right. Evaluating the colour matrix elements and performing the integrals over transverse momenta we confirm the result quoted in eq. 2.21).

Again we can do a Feynman diagram decomposition, see figure 3:

$$
\begin{aligned}
& \sigma_{1, \text { out }=\text { second-hardest }}=2^{5}\left(-\frac{2 \alpha_{s}}{\pi}\right)^{4} \int_{Q_{0}}^{Q} \frac{d k_{T}}{k_{T}}\left(\ln \frac{Q}{k_{T}}\right) \int_{k_{T}}^{Q} \frac{d k_{T}^{\prime}}{k_{T}^{\prime}}\left(\int_{Q_{0}}^{k_{T}} \frac{d k_{T}^{\prime \prime}}{k_{T}^{\prime \prime}}\right)^{2} \\
& \times\left\langle m_{0}\right|(\left(-\frac{1}{2} Y \mathbf{t}_{1} \cdot \mathbf{t}_{4}\right)\left(+\frac{1}{2} i \pi \mathbf{t}_{1} \cdot \mathbf{t}_{2}\right) \\
&\left.+\left(-\frac{1}{2} i \pi \mathbf{t}_{1} \cdot \mathbf{t}_{2}\right)\left(-\frac{1}{2} Y \mathbf{t}_{1} \cdot \mathbf{t}_{4}\right)\right) 2\left(\frac{1}{2} \mathbf{t}_{1}^{2}\right)\left(+\frac{1}{2} i \pi \mathbf{t}_{1} \cdot \mathbf{t}_{2}\right) \\
&-2\left(\frac{1}{2} \mathbf{t}_{1}^{a \dagger}\right)\left(\left(-\frac{1}{2} Y \mathbf{T}_{1} \cdot \mathbf{T}_{4}\right)\left(+\frac{1}{2} i \pi \mathbf{T}_{1} \cdot \mathbf{T}_{2}\right)\right. \\
&\left.+\left(-\frac{1}{2} i \pi \mathbf{T}_{1} \cdot \mathbf{T}_{2}\right)\left(-\frac{1}{2} Y \mathbf{T}_{1} \cdot \mathbf{T}_{4}\right)\right) \mathbf{t}_{1}^{a}\left(+\frac{1}{2} i \pi \mathbf{t}_{1} \cdot \mathbf{t}_{2}\right)\left|m_{0}\right\rangle
\end{aligned}
$$

The first two terms in the matrix element correspond to graphs (a) and (c) whilst the latter pair correspond to graphs (b) and (d). Again $\mathbf{t}_{1}^{2}$ (and $\mathbf{t}_{1}^{a \dagger} \cdots \mathbf{t}_{1}^{a}$ ) is shorthand for the sum 


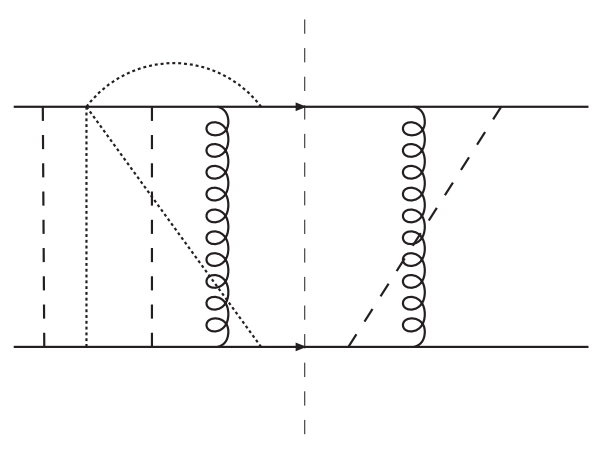

(a)

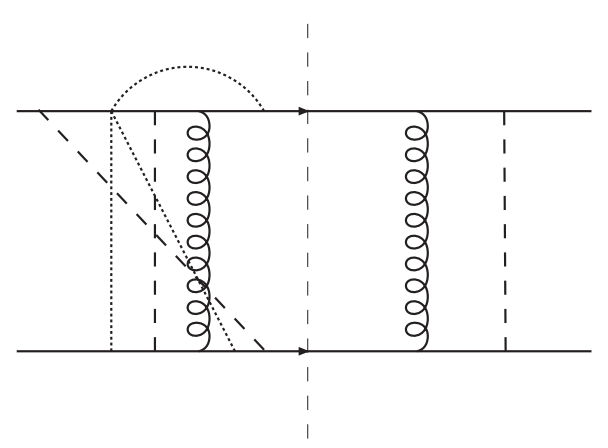

(c)

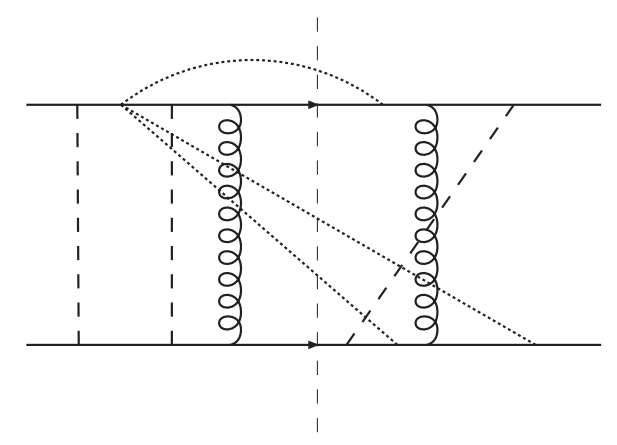

(b)

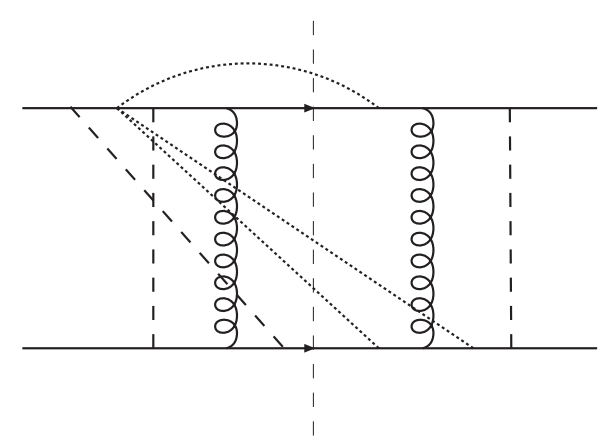

(d)

Figure 3: The relevant Feynman diagrams in the case that the out-of-gap (dotted) gluon is the second hardest gluon. The dashed lines indicate soft (eikonal and Coulomb) gluons. Each subfigure represents three Feynman diagrams, corresponding to the three different ways of attaching the outof-gap gluon. The soft gluon to the right of the cut should only be integrated over the region in which it has transverse momentum less than the out-of-gap gluon.

over graphs where the out-of-gap real gluon connects parton 1 with partons 2,3 and 4 , and we do not show the additional $3 \times 4$ graphs that occur when the out-of-gap gluon couples to parton 1 on the right of the cut.

\section{Results}

Our main results are eqs. (4.18) and (4.20), or equivalently the Feynman diagrams in figures 2 and 3 . In the case that the scattered particles are quarks, one can check that they reproduce the results given in [7] and section 2,

$$
\begin{aligned}
\sigma_{1, \text { out }=\text { hardest }, q q} & =-\sigma_{0}\left(\frac{2 \alpha_{s}}{\pi}\right)^{4} \ln ^{5}\left(\frac{Q}{Q_{0}}\right) \pi^{2} Y \frac{N^{2}-2}{240}, \\
\sigma_{1, \text { out }=\text { second-hardest }, q q} & =-\sigma_{0}\left(\frac{2 \alpha_{s}}{\pi}\right)^{4} \ln ^{5}\left(\frac{Q}{Q_{0}}\right) \pi^{2} Y \frac{N^{2}-1}{120} \\
\sigma_{1, q q} & =-\sigma_{0}\left(\frac{2 \alpha_{s}}{\pi}\right)^{4} \ln ^{5}\left(\frac{Q}{Q_{0}}\right) \pi^{2} Y \frac{3 N^{2}-4}{240} .
\end{aligned}
$$


The simplicity of the results in colour basis independent notation also allows us to calculate the coefficient of the super-leading logarithm for other scattering processes, in particular involving gluons. The colour algebra is no more complicated in principle than for quarks, and we have checked our results using the Colour program [28]. For quark-gluon scattering we obtain

$$
\begin{aligned}
\sigma_{1, \text { out }=\text { hardest }, q g} & =-\sigma_{0}\left(\frac{2 \alpha_{s}}{\pi}\right)^{4} \ln ^{5}\left(\frac{Q}{Q_{0}}\right) \pi^{2} Y \frac{N^{2}}{80} \\
\sigma_{1, \text { out }=\text { second-hardest }, q g} & =-\sigma_{0}\left(\frac{2 \alpha_{s}}{\pi}\right)^{4} \ln ^{5}\left(\frac{Q}{Q_{0}}\right) \pi^{2} Y \frac{N^{2}}{60} \\
\sigma_{1, q g} & =-\sigma_{0}\left(\frac{2 \alpha_{s}}{\pi}\right)^{4} \ln ^{5}\left(\frac{Q}{Q_{0}}\right) \pi^{2} Y \frac{7 N^{2}}{240} .
\end{aligned}
$$

This result is remarkable in that it does not depend upon whether the out-of-gap gluon is collinear to the quark or to the gluon. For gluon-gluon scattering we find

$$
\begin{aligned}
\sigma_{1, \text { out }=\text { hardest }, g g} & =-\sigma_{0}\left(\frac{2 \alpha_{s}}{\pi}\right)^{4} \ln ^{5}\left(\frac{Q}{Q_{0}}\right) \pi^{2} Y \frac{5 N^{2}+12}{240}, \\
\sigma_{1, \text { out }=\text { second-hardest }, g g} & =-\sigma_{0}\left(\frac{2 \alpha_{s}}{\pi}\right)^{4} \ln ^{5}\left(\frac{Q}{Q_{0}}\right) \pi^{2} Y \frac{N^{2}}{60}, \\
\sigma_{1, g g} & =-\sigma_{0}\left(\frac{2 \alpha_{s}}{\pi}\right)^{4} \ln ^{5}\left(\frac{Q}{Q_{0}}\right) \pi^{2} Y \frac{3 N^{2}+4}{80} .
\end{aligned}
$$

Note that in all cases $\sigma_{0}$ is the corresponding Born level cross-section (i.e. it differs by a colour factor for each sub-process). The results for processes involving anti-quarks are identical to the corresponding processes involving quarks.

\section{Outlook}

In this paper we have reconsidered the super-leading logarithms discovered for gaps between jets observables in [7] using a colour basis independent notation. It has added considerable insight, allowing their calculation to be condensed down to a small number of Feynman diagrams (figures 2 and 3) and allowing the first super-leading logarithm to be calculated for gluon scattering processes. The main questions still remain open however: the structure of higher order super-leading logarithms; how widespread they are in other observables for hadron collisions; and whether they can be reorganized and resummed or removed by a suitable redefinition of observables or of incoming partonic states. We hope that the insight provided by the colour basis independent notation will ultimately help to illuminate these questions.

We close by noting that further simplifications are possible. In particular, the commutators between gluon exchanges from an external leg in different orders can be written as emission off the exchanged Coulomb gluons. The colour matrix elements in eqs. (4.18) and $(4.20)$ can then be shown to be identical to those illustrated in figure 1 . In this form it is clear that the superleading logarithms arise as a result of the non-Abelian nature of 

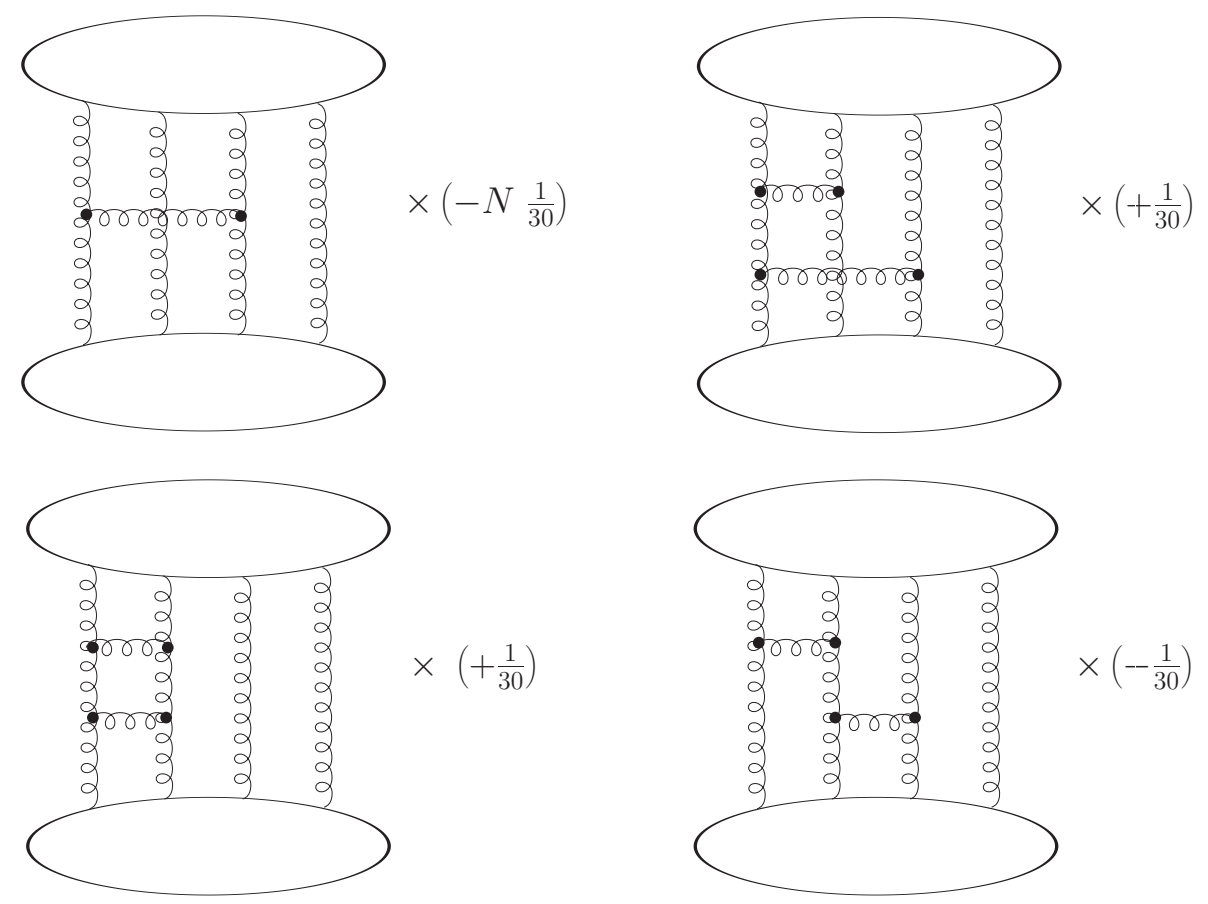

Figure 4: The four diagrams that generate the colour matrix elements when the out-of-gap gluon is the hardest gluon. In the case that the out-of-gap gluon is next-to-hardest, only the first diagram contributes. The upper and lower loops can be quarks, anti-quarks or gluons. Note that these are not Feynman diagrams or even uncut diagrams; they represent only the colour factor of the final result. In the first diagram one of the original six gluon lines has been contracted away, resulting in the additional factor of $N$.

the Coulomb gluon interaction. It is also now clear why the coefficient of the superleading logarithm is independent of whether the out-of-gap gluon is collinear to parton 1 or 2: the result is invariant under interchange of the particle types in the upper and lower loops.

\section{Acknowledgments}

We are grateful to Stefano Catani for suggesting the colour basis independent notation used here. This work was begun at the Galileo Galilei Institute for Theoretical Physics workshop entitled "Advancing Collider Physics: from Twistors to Monte Carlos"; MHS gratefully acknowledges the Institute's financial support. We also thank Mrinal Dasgupta, James Keates, Malin Sjödahl and George Sterman for interesting discussions of these and related topics.

\section{References}

[1] N. Kidonakis, G. Oderda and G. Sterman, Evolution of color exchange in QCD hard scattering, Nucl. Phys. B 531 (1998) 365 hep-ph/9803241.

[2] G. Oderda and G. Sterman, Energy and color flow in dijet rapidity gaps, Phys. Rev. Lett. 81 (1998) 3591 hep-ph/9806530. 
[3] G. Oderda, Dijet rapidity gaps in photoproduction from perturbative QCD, Phys. Rev. D 61 (2000) 014004 hep-ph/9903240.

[4] C.F. Berger, T. Kucs and G. Sterman, Energy flow in interjet radiation, Phys. Rev. D 65 (2002) 094031 hep-ph/0110004.

[5] R.B. Appleby and M.H. Seymour, The resummation of inter-jet energy flow for gaps-between-jets processes at HERA, JHEP 09 (2003) 056 hep-ph/0308086.

[6] J.R. Forshaw, A. Kyrieleis and M.H. Seymour, Gaps between jets in the high energy limit, JHEP 06 (2005) 034 hep-ph/0502086.

[7] J.R. Forshaw, A. Kyrieleis and M.H. Seymour, Super-leading logarithms in non-global observables in QCD, JHEP 08 (2006) 059 hep-ph/0604094.

[8] M. Dasgupta and G.P. Salam, Resummation of non-global QCD observables, Phys. Lett. B 512 (2001) 323 hep-ph/0104277.

[9] M. Dasgupta and G.P. Salam, Accounting for coherence in interjet E(t) flow: a case study, JHEP 03 (2002) 017 hep-ph/0203009.

[10] R.B. Appleby and M.H. Seymour, Non-global logarithms in inter-jet energy flow with kt clustering requirement, JHEP 12 (2002) 063 hep-ph/0211426.

[11] A. Kyrieleis, J.R. Forshaw and M.H. Seymour, Breakdown of QCD coherence?, PoS (DIFF2006) 031 hep-ph/0612202.

[12] M.H. Seymour, Breakdown of coherence?, talk given at 12th International Conference on Elastic and Diffractive Scattering: Forward Physics and QCD, Hamburg, DESY, Germany, 21-25 May 2007, arXiv:0710.2733.

[13] J. Collins and J.-W. Qiu, $k_{T}$ factorization is violated in production of hightransverse-momentum particles in hadron-hadron collisions, Phys. Rev. D 75 (2007) 114014 arXiv:0705.2141.

[14] J. Collins, 2-soft-gluon exchange and factorization breaking, arXiv:0708.4410.

[15] J. Keates, work in progress, private communication.

[16] S. Catani, M. Ciafaloni and G. Marchesini, Noncancelling infrared divergences in QCD coherent state, Nucl. Phys. B 264 (1986) 588.

[17] M.H. Seymour, Symmetry of anomalous dimension matrices for colour evolution of hard scattering processes, JHEP 10 (2005) 029 hep-ph/0508305.

[18] M.H. Seymour and M. Sjödahl, in preparation.

[19] A. Kyrieleis and M.H. Seymour, The colour evolution of the process $q q \rightarrow$ qqg, JHEP 01 (2006) 085 hep-ph/0510089.

[20] S. Catani and M.H. Seymour, The dipole formalism for the calculation of QCD jet cross sections at next-to-leading order, Phys. Lett. B 378 (1996) 287 hep-ph/9602277.

[21] S. Catani and M.H. Seymour, A general algorithm for calculating jet cross sections in NLO QCD, Nucl. Phys. B 485 (1997) 291 [Erratum ibid. B 510 (1998) 503] hep-ph/9605323].

[22] Y.L. Dokshitzer and G. Marchesini, Soft gluons at large angles in hadron collisions, JHEP 01 (2006) 007 hep-ph/0509078. 
[23] Y.L. Dokshitzer and G. Marchesini, Hadron collisions and the fifth form factor, Phys. Lett. B 631 (2005) 118 hep-ph/0508130.

[24] J.C. Collins, D.E. Soper and G. Sterman, Factorization for short distance hadron-hadron scattering, Nucl. Phys. B 261 (1985) 104.

[25] J.C. Collins, D.E. Soper and G. Sterman, Soft gluons and factorization, Nucl. Phys. B 308 (1988) 833 .

[26] J.C. Collins, D.E. Soper and G. Sterman, Factorization is not violated, Phys. Lett. B 438 (1998) 184 hep-ph/9806234.

[27] J.R. Forshaw and M. Sjodahl, Soft gluons in Higgs plus two jet production, JHEP 09 (2007) 119 arXiv:0705.1504.

[28] J. Hakkinen and H. Kharraziha, Colour: a computer program for QCD colour factor calculations, Comput. Phys. Commun. 100 (1997) 311 [hep-ph/9603229]. 